Supporting Information for

\title{
Structural Changes of the Active Center during the Photoactivation of
}

\section{Xenopus (6-4) Photolyase ${ }^{\dagger}$}

Daichi Yamada, $₫$ Junpei Yamamoto, $§$ Yu Zhang, $₫$ Tatsuya Iwata, $₫$ Kenichi Hitomi,,,$\perp$

Elizabeth D. Getzoff, $\|$ Shigenori Iwai,$\S$ and Hideki Kandori*, $\$$

†Department of Frontier Materials, Nagoya Institute of Technology, Showa-ku, Nagoya 4668555, Japan, §Graduate School of Engineering Science, Osaka University, Toyonaka, Osaka 5608531, Japan, |Department of Molecular Biology and the Skaggs Institute for Chemical Biology,

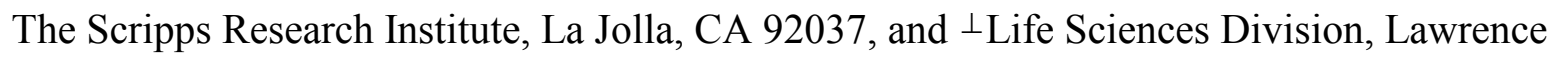
Berkeley National Laboratory, Berkeley, CA 94720, USA, 


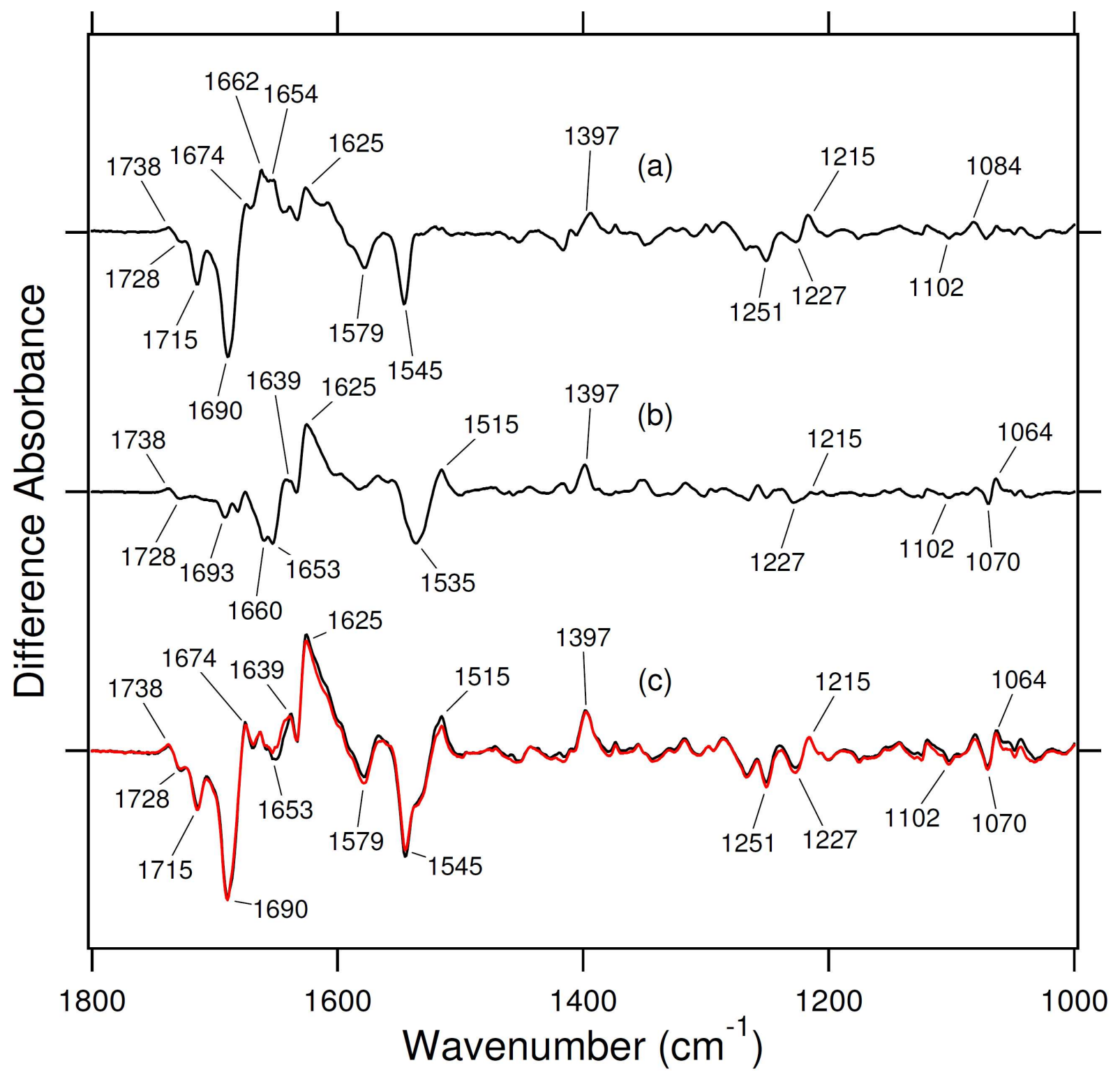

Figure S1. Light-induced difference FTIR spectra of Xenopus (6-4) PHR in the presence of (6-4) PP at $277 \mathrm{~K}$. (a) Difference spectrum generated by $450 \mathrm{~nm}$ of light illumination (an interference filter) of $\mathrm{FAD}^{\text {ox }}$ for $4 \mathrm{~min}$, where the product consists of $49 \% \mathrm{FADH}^{\bullet}$ and $51 \%$ $\mathrm{FADH}^{-}{ }^{1}$ (b) After the illumination for panel a, the sample was illuminated with $>550 \mathrm{~nm}$ light for 1 min, which provided the $\mathrm{FADH}^{-}$minus $\mathrm{FADH}^{\bullet}$ difference spectrum. (c) Sum of spectra $\mathrm{a}$ and $\mathrm{b}$ (red line) that coincides with the $\mathrm{FADH}^{-}$minus $\mathrm{FAD}^{\mathrm{ox}}$ difference spectrum obtained by illumination of $\mathrm{FAD}^{\mathrm{ox}}$ with $>450 \mathrm{~nm}$ light for 4 min (black line). One division of the $y$-axis corresponds to 0.008 absorbance units. 


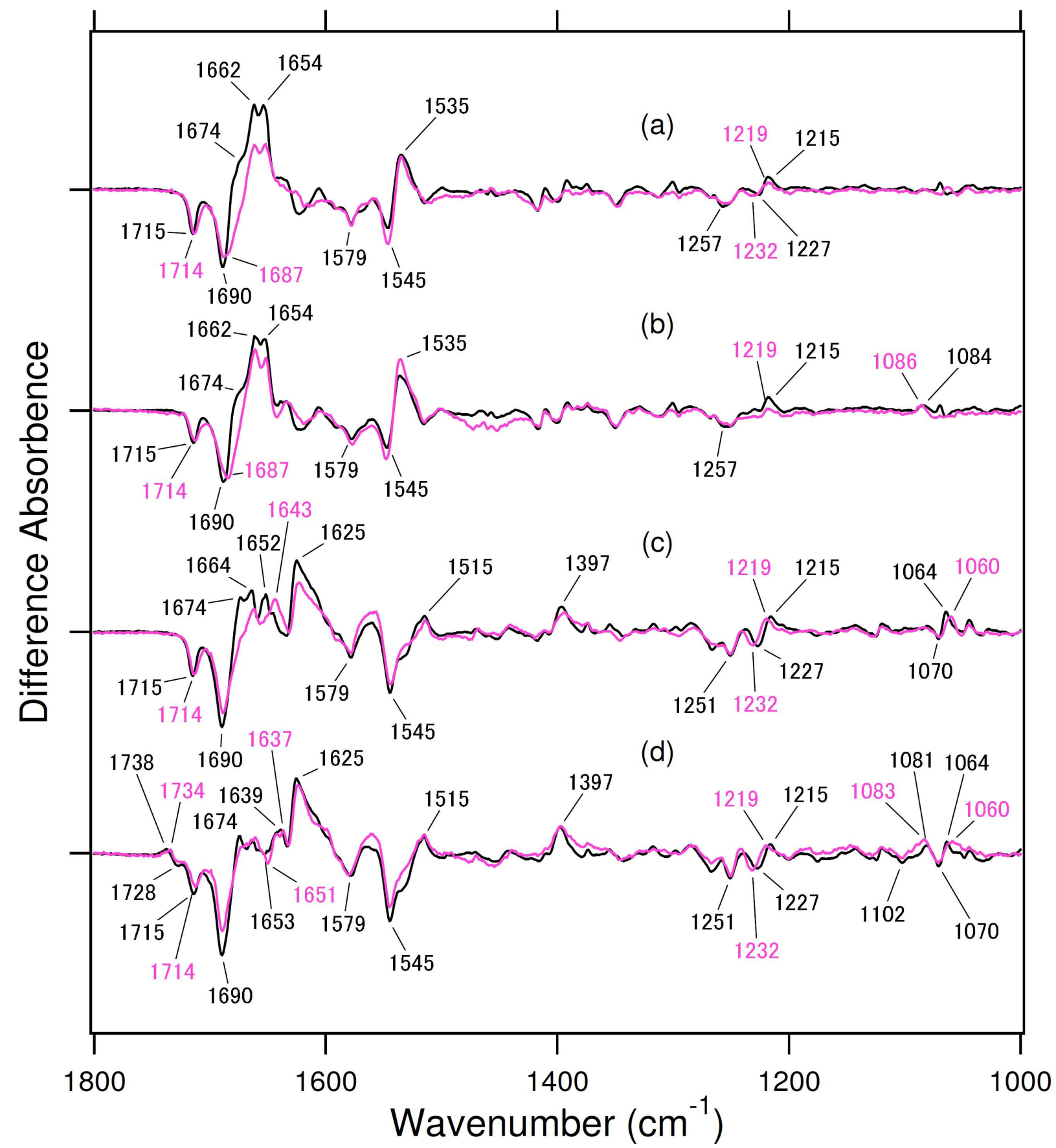

Figure S2. Comparison of difference FTIR spectra of Xenopus (6-4) PHR in $\mathrm{H}_{2} \mathrm{O}$ (black line) and $\mathrm{D}_{2} \mathrm{O}$ (purple line). (a) $\mathrm{FADH}^{\bullet}$ minus FAD ${ }^{\text {ox }}$ difference FTIR spectra without (6-4) PP. (b) $\mathrm{FADH}^{\bullet}$ minus $\mathrm{FAD}^{\mathrm{ox}}$ difference FTIR spectra with (6-4) PP. (c) $\mathrm{FADH}^{-}$minus $\mathrm{FAD}^{\text {ox }}$ difference FTIR spectra without (6-4) PP. (d) $\mathrm{FADH}^{-}$minus $\mathrm{FAD}^{\text {ox }}$ difference FTIR spectra with (6-4) PP. One division of the $y$-axis corresponds to 0.01 absorbance units. 


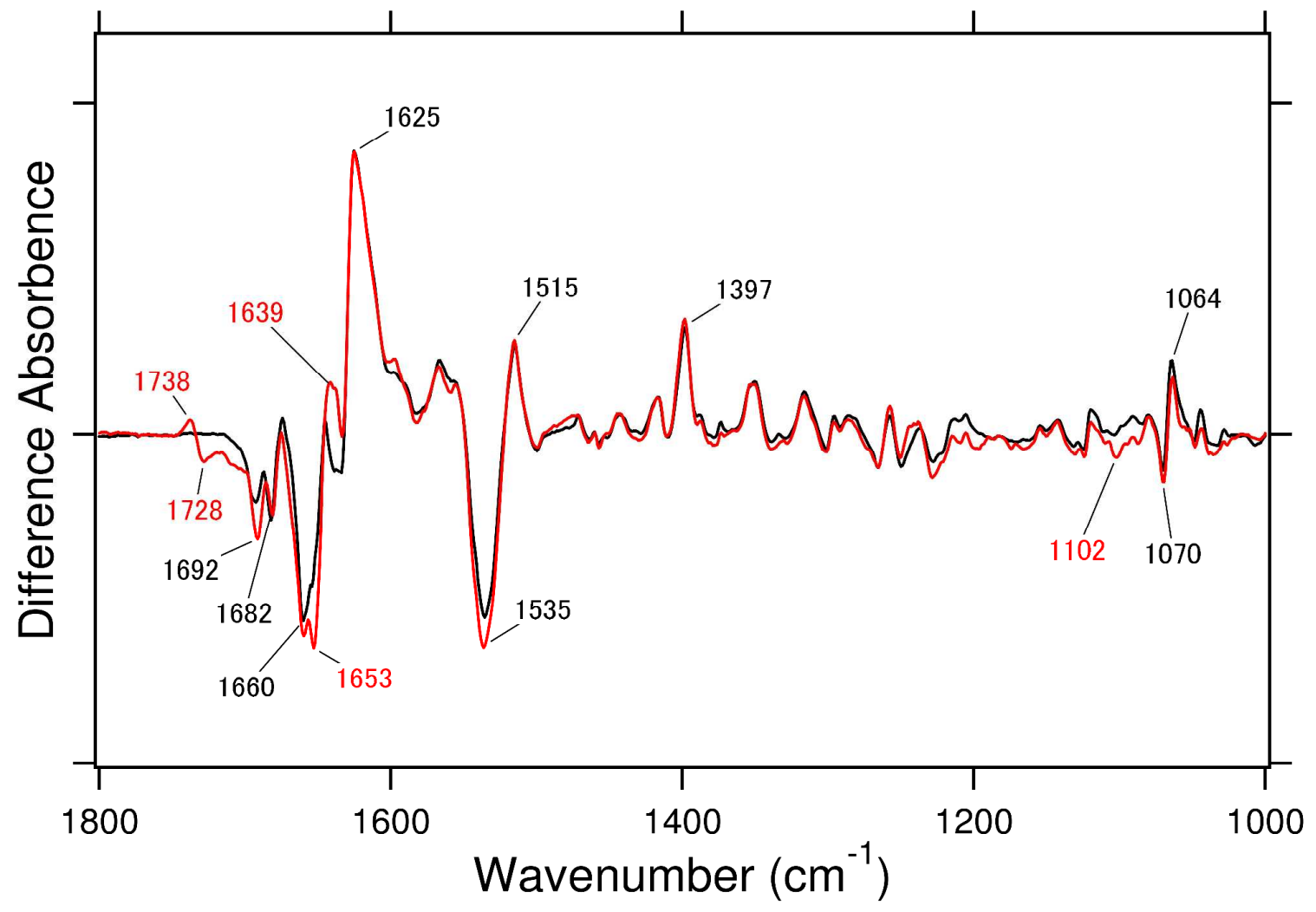

Figure S3. Light-induced difference FTIR spectra of (6-4) PHR in the change from FADH ${ }^{\bullet}$ to $\mathrm{FADH}^{-}$without (black line) and with (red line) (6-4) PP at $277 \mathrm{~K}$. Black and red lines were reproduced from a previous study ${ }^{1}$ and Figure $\mathrm{S} 1 \mathrm{~b}$. One division of the $y$-axis corresponds to 0.005 absorbance units. 


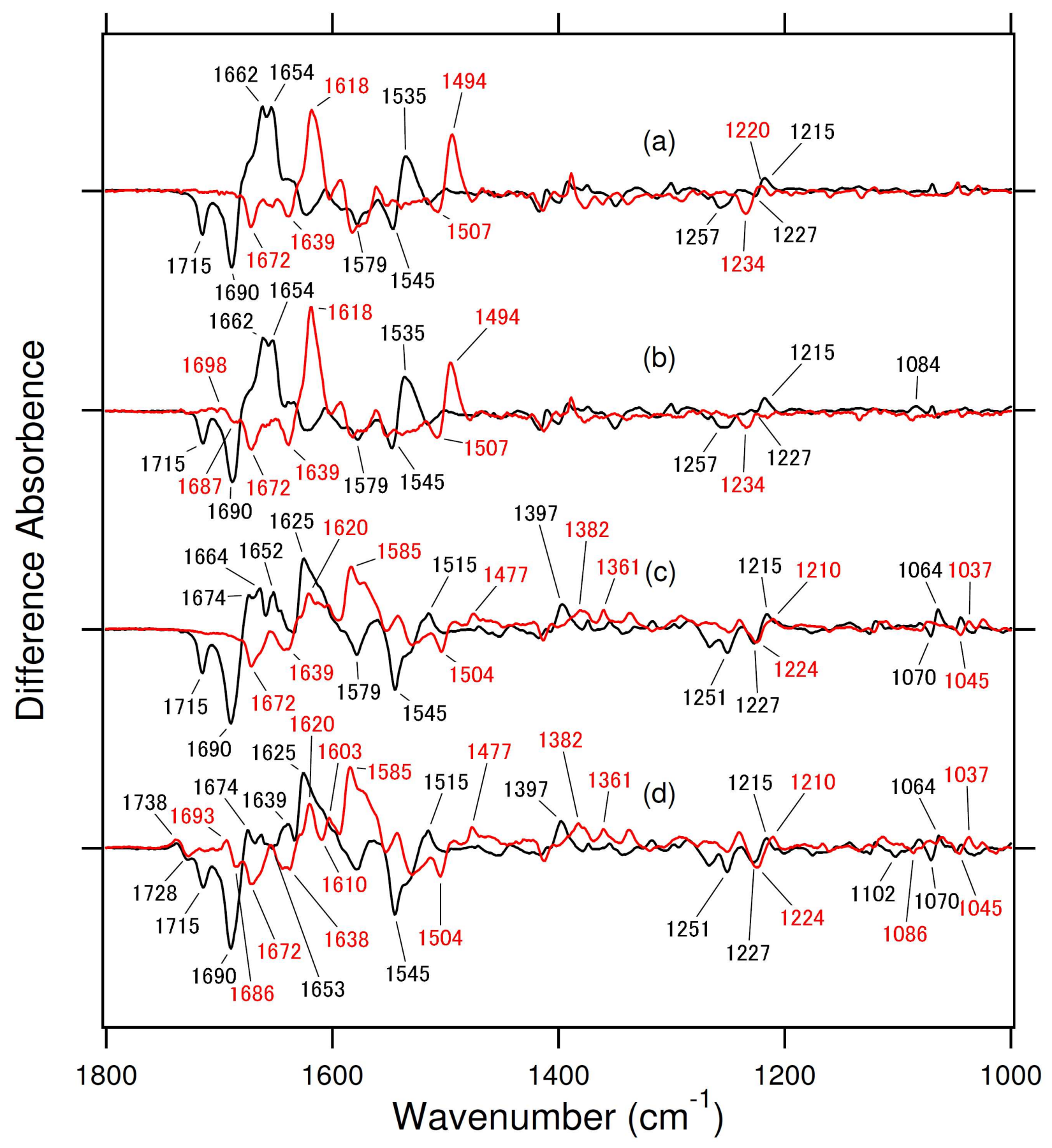

Figure S4. Comparison of difference FTIR spectra of unlabeled (black line) and ${ }^{13} \mathrm{C}$-labeled (red line) Xenopus (6-4) PHR. (a) FADH' minus FAD ${ }^{\circ}$ difference FTIR spectra without (6-4) PP. (b) $\mathrm{FADH}^{\bullet}$ minus $\mathrm{FAD}^{\mathrm{ox}}$ difference FTIR spectra with (6-4) PP. (c) $\mathrm{FADH}^{-}$minus $\mathrm{FAD}^{\text {ox }}$ difference FTIR spectra without (6-4) PP. (d) $\mathrm{FADH}^{-}$minus FAD ${ }^{\text {ox }}$ difference FTIR spectra with (6-4) PP. One division of the $y$-axis corresponds to 0.01 absorbance units. 


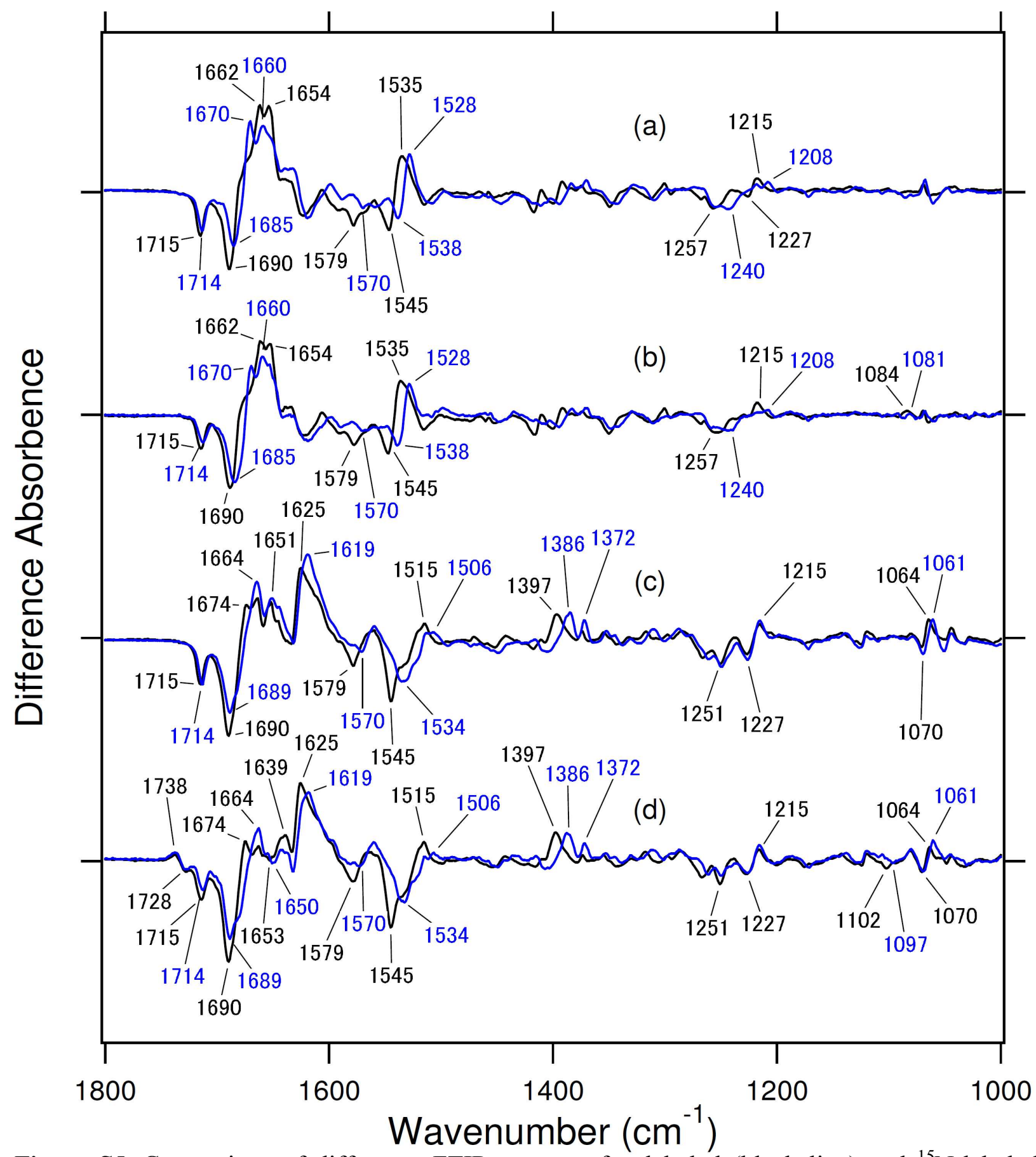

Figure S5. Comparison of difference FTIR spectra of unlabeled (black line) and ${ }^{15} \mathrm{~N}$-labeled (blue line) Xenopus (6-4) PHR. (a) FADH• minus FAD ${ }^{\circ}$ difference FTIR spectra without (6-4) PP. (b) $\mathrm{FADH}^{\bullet}$ minus $\mathrm{FAD}^{\mathrm{ox}}$ difference FTIR spectra with (6-4) PP. (c) $\mathrm{FADH}^{-}$minus $\mathrm{FAD}^{\text {ox }}$ difference FTIR spectra without (6-4) PP. (d) $\mathrm{FADH}^{-}$minus FAD ${ }^{\text {ox }}$ difference FTIR spectra with (6-4) PP. One division of the $y$-axis corresponds to 0.01 absorbance units. 


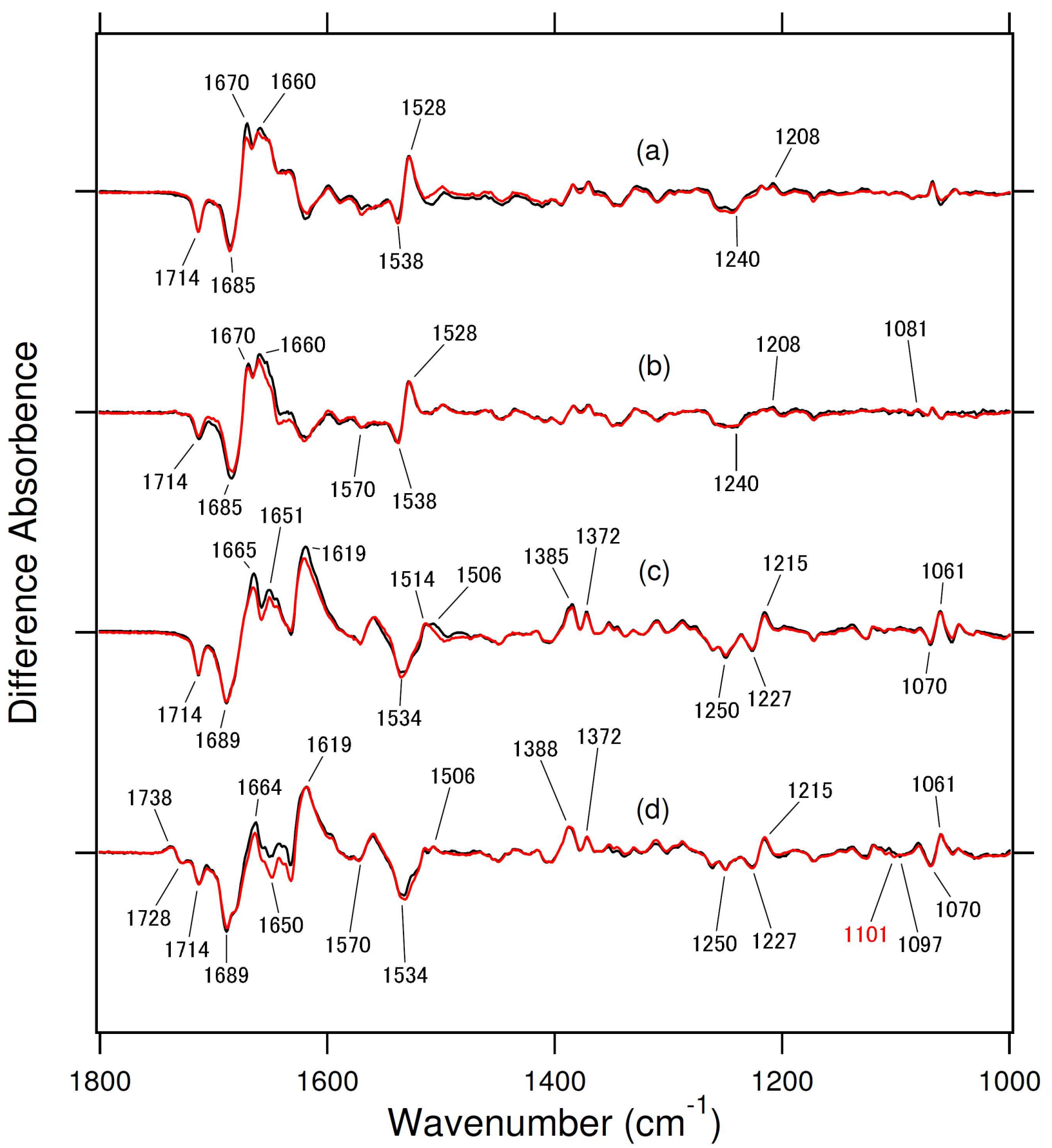

Figure S6. Comparison of difference FTIR spectra of ${ }^{15} \mathrm{~N}$-labeled (black line) and ${ }^{14} \mathrm{~N}$ His $/{ }^{15} \mathrm{~N}$-labeled (red line) Xenopus (6-4) PHR. (a) FADH• minus FAD ${ }^{\text {ox }}$ difference FTIR spectra without (6-4) PP. (b) FADH ${ }^{\bullet}$ minus FAD ${ }^{\text {ox }}$ difference FTIR spectra with (6-4) PP. (c) $\mathrm{FADH}^{-}$minus $\mathrm{FAD}^{\text {ox }}$ difference FTIR spectra without (6-4) PP. (d) $\mathrm{FADH}^{-}$minus FAD $^{\text {ox }}$ difference FTIR spectra with (6-4) PP. One division of the $y$-axis corresponds to 0.01 absorbance units. 
(a)

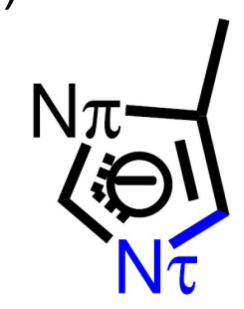

(b)

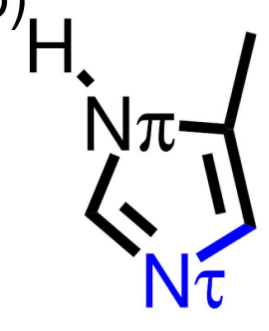

(c)

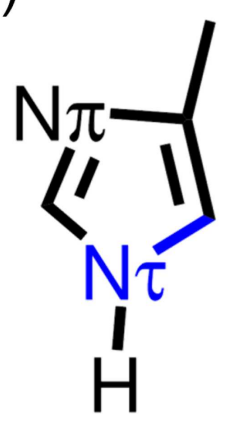

(d)

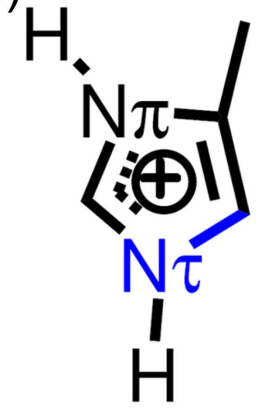

Figure S7. Structural diagram of a His side chain: (a) imidazolate form, (b) N $\pi$-protonated form, (c) $\mathrm{N} \tau$-protonated form and (d) imidazolium form. 


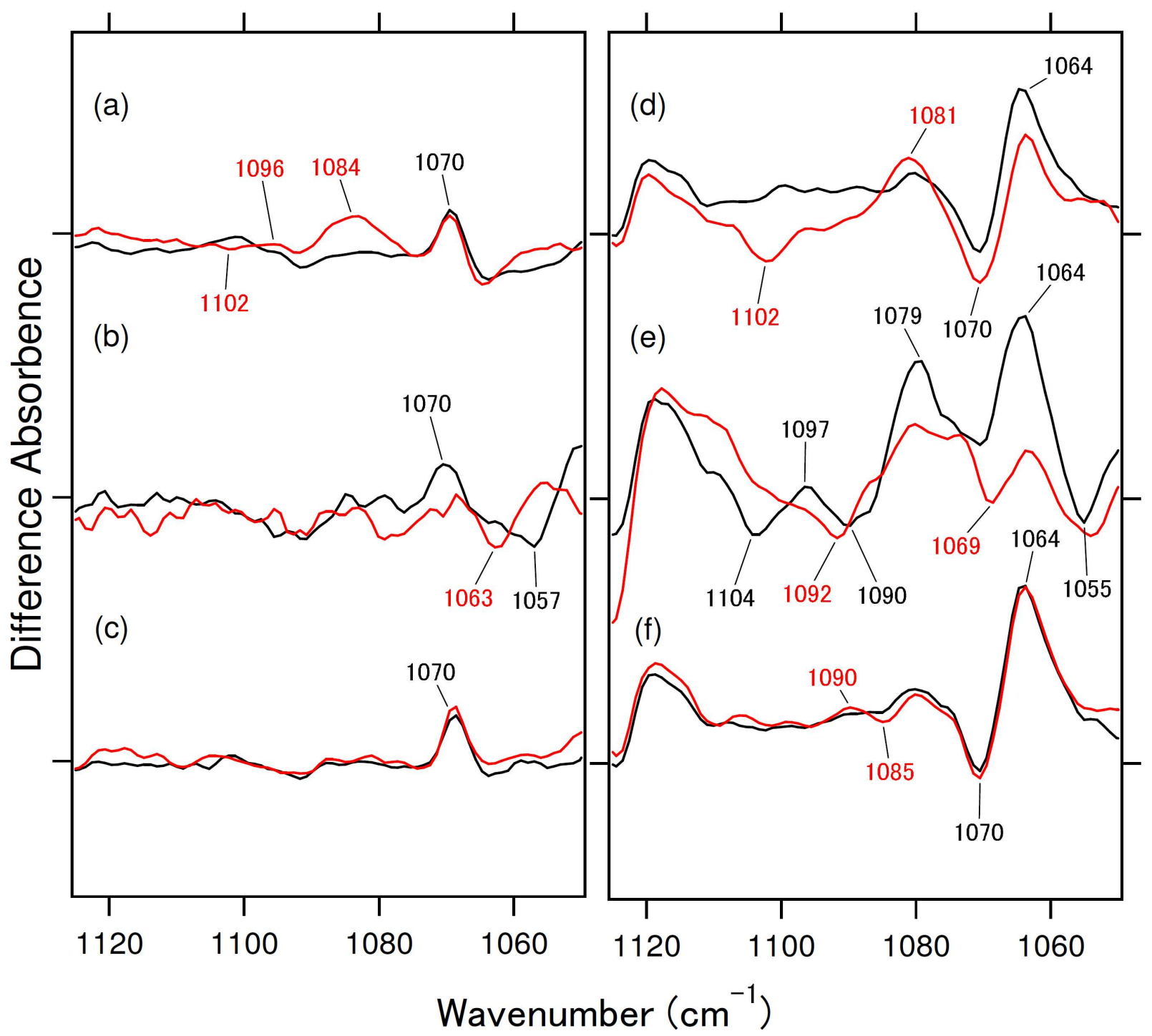

Figure S8. Impact of mutations on difference FTIR spectra with (red line) and without (black line) (6-4) PP in the 1125-1050 $\mathrm{cm}^{-1}$ range. FADH ${ }^{\bullet}$ minus $\mathrm{FAD}^{\mathrm{ox}}$ spectra of wild-type (a), H354A (b) and H358A mutants (c) of (6-4) PHR. FADH ${ }^{-}$minus FAD ${ }^{\text {ox }}$ spectra of wild-type (d), H354A (e) and H358A mutants (f) of (6-4) PHR. One division of the $y$-axis corresponds to 0.002 absorbance units. 


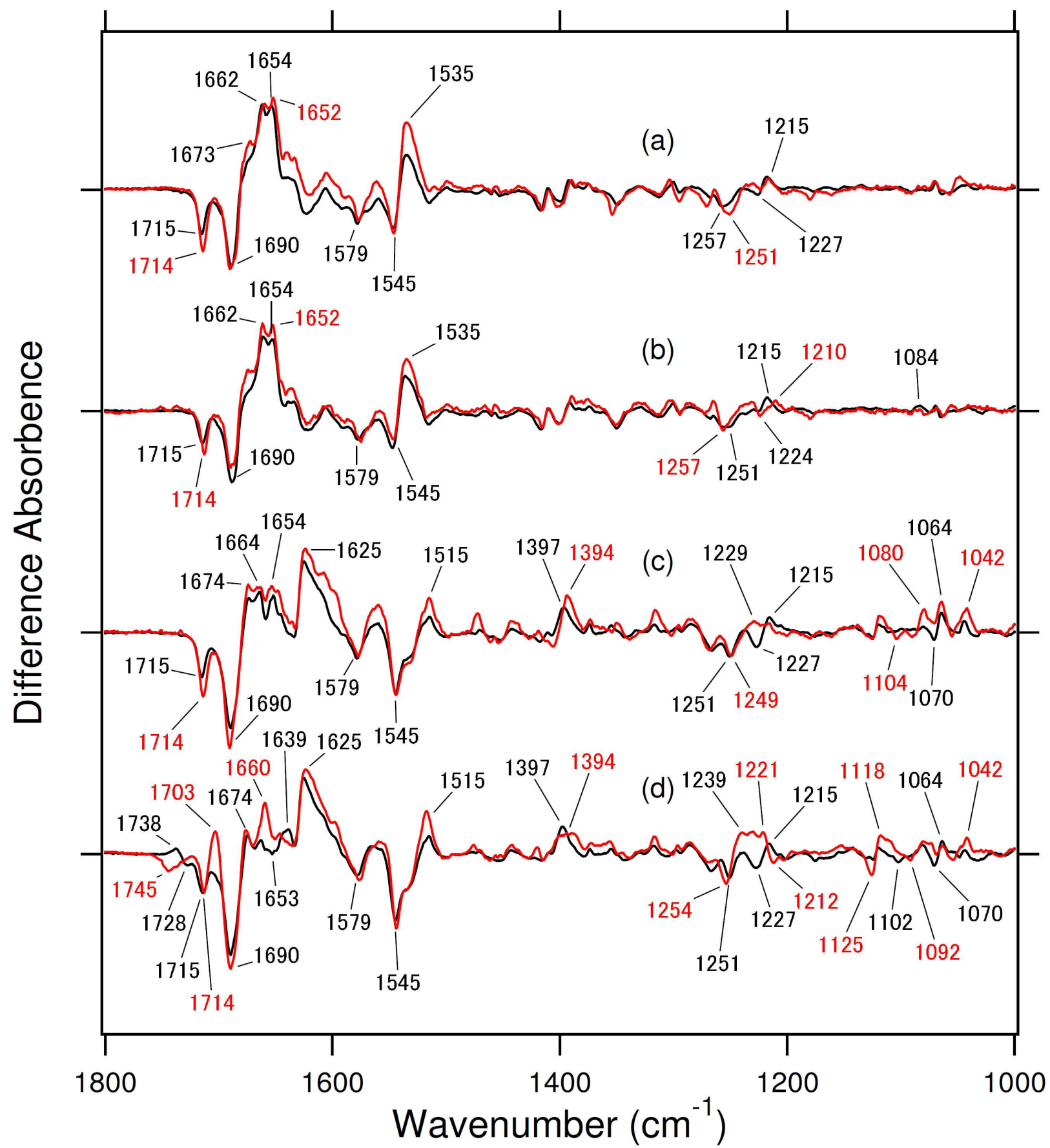

Figure S9. Comparison of difference FTIR spectra of WT (black line) and H354A mutant (red line) of Xenopus (6-4) PHR. (a) FADH minus FAD ${ }^{\circ}$ difference FTIR spectra without (6-4) PP. (b) $\mathrm{FADH}^{\bullet}$ minus FAD ${ }^{\text {ox }}$ difference FTIR spectra with (6-4) PP. (c) FADH $\mathrm{FH}^{-}$minus FAD ${ }^{\text {ox }}$ difference FTIR spectra without (6-4) PP. (d) FADH ${ }^{-}$minus FAD ox difference FTIR spectra with (6-4) PP. One division of the $y$-axis corresponds to 0.01 absorbance units. 


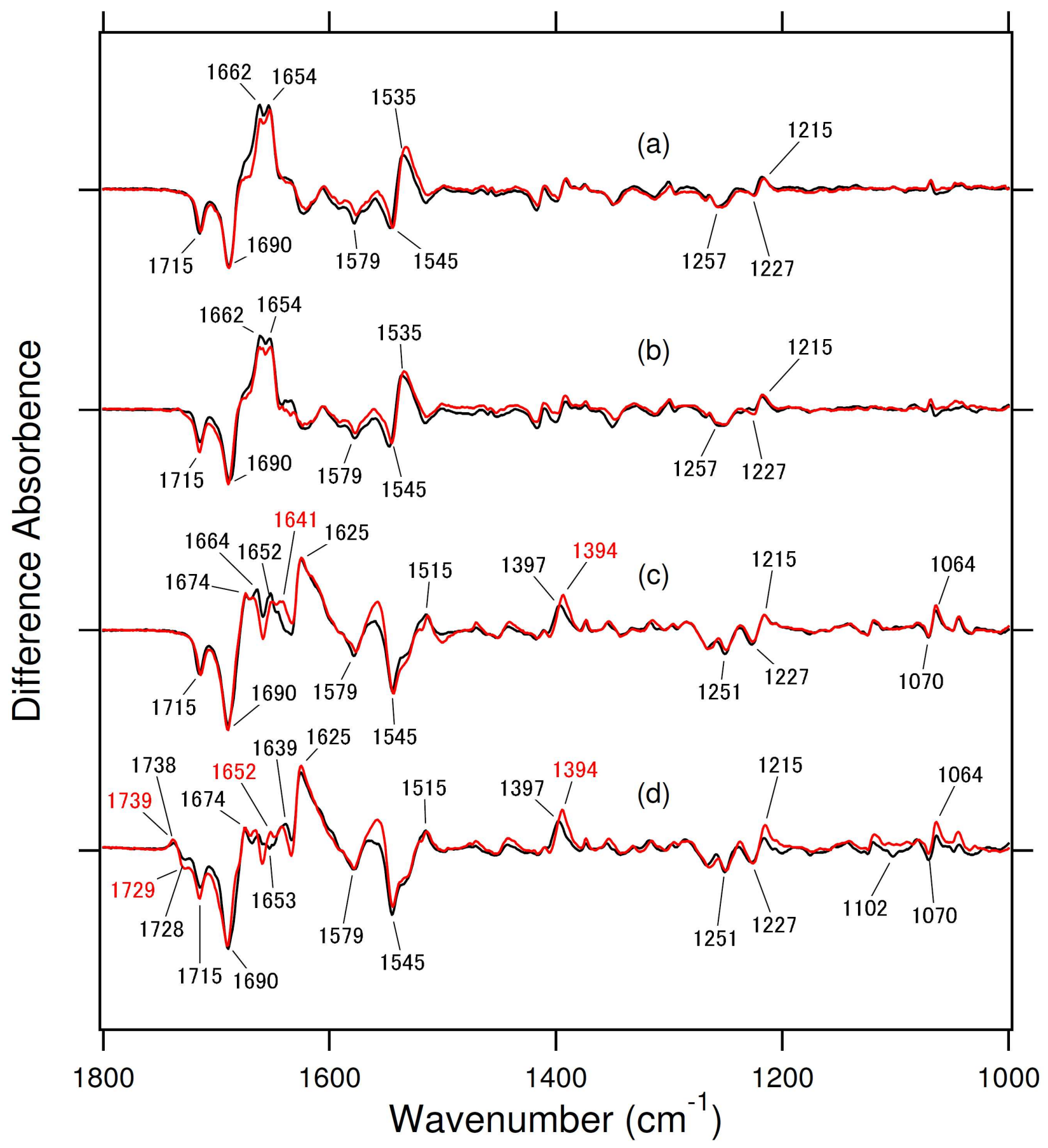

Figure S10. Comparison of difference FTIR spectra of WT (black line) and H358A mutant (red line) of Xenopus (6-4) PHR. (a) FADH ${ }^{\bullet}$ minus FAD ${ }^{\text {ox }}$ difference FTIR spectra without (6-4) PP. (b) FADH ${ }^{\bullet}$ minus FAD ${ }^{\text {ox }}$ difference FTIR spectra with (6-4) PP. (c) $\mathrm{FADH}^{-}$minus FAD ${ }^{\text {ox }}$ difference FTIR spectra without (6-4) PP. (d) FADH ${ }^{-}$minus FAD ${ }^{\text {ox }}$ difference FTIR spectra with (6-4) PP. One division of the $y$-axis corresponds to 0.01 absorbance units. 


\section{REFERENCES}

(1) Yamada, D., Zhang, Y., Iwata, T., Hitomi, K., Getzoff, E. D., and Kandori, H. (2012) Fouriertransform infrared study of the photoactivation process of Xenopus (6-4) photolyase. Biochemistry 51, 5774-5783. 